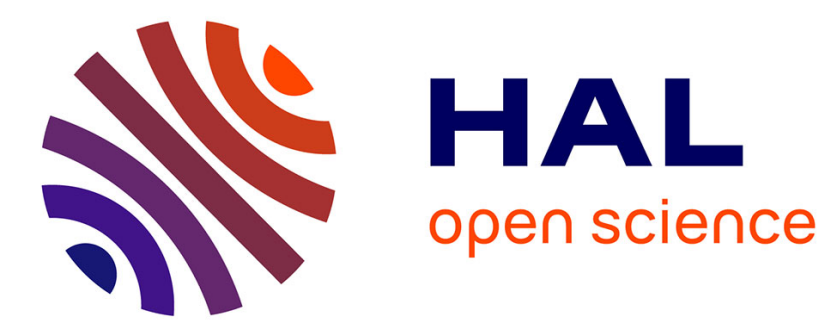

\title{
MMS Observations of Multiscale Hall Physics in the Magnetotail
}

\author{
Love Alm, Mats André, Daniel B Graham, Yuri Khotyaintsev, Andris \\ Vaivads, Charles R Chappell, Jérémy Dargent, Stephen Fuselier, Stein \\ Haaland, Benoit Lavraud, et al.
}

\section{To cite this version:}

Love Alm, Mats André, Daniel B Graham, Yuri Khotyaintsev, Andris Vaivads, et al.. MMS Observations of Multiscale Hall Physics in the Magnetotail. Geophysical Research Letters, In press, 10.1029/2019GL084137 . hal-02372728

\section{HAL Id: hal-02372728 \\ https://hal.science/hal-02372728}

Submitted on 20 Nov 2019

HAL is a multi-disciplinary open access archive for the deposit and dissemination of scientific research documents, whether they are published or not. The documents may come from teaching and research institutions in France or abroad, or from public or private research centers.
L'archive ouverte pluridisciplinaire HAL, est destinée au dépôt et à la diffusion de documents scientifiques de niveau recherche, publiés ou non, émanant des établissements d'enseignement et de recherche français ou étrangers, des laboratoires publics ou privés. 


\section{Geophysical Research Letters}

\author{
RESEARCH LETTER \\ 10.1029/2019GL084137 \\ Key Points: \\ - MMS multispacecraft observation \\ of Hall electric fields during a deep \\ partial plasma sheet crossing \\ - Mixing of hot (20 keV) and \\ moderately cold $(1.5 \mathrm{keV})$ ions \\ generate multiscale Hall electric \\ fields \\ - Electron and cold/hot ion \\ demagnetization boundaries are \\ observed near their predicted \\ locations
}

Correspondence to:

L. Alm,

love.alm@irfu.se

Citation:

Alm, L., Andr'e, M., Graham, D. B., Khotyaintsev, Y. V., Vaivads, A., Chappel, C. R., et al. (2019). MMS observations of multiscale Hall physics in the magnetotail. Geophysical Research Letters, 46, 10,230-10,239. https://doi.org/10.1029/2019GL084137

Received 18 JUN 2019 Accepted 26 AUG 2019 Accepted article online 29 AUG 2019 Published online 5 SEP 2019

C2019. American Geophysical Union. All Rights Reserved.

\section{MMS Observations of Multiscale Hall Physics in the Magnetotail}

\author{
Love Alm ${ }^{1}$ (D), Mats André1 (iD), Daniel B. Graham ${ }^{1}$ (D), Yuri V. Khotyaintsev' ${ }^{1}$, Andris Vaivads ${ }^{2}$ (iD), \\ Charles. R. Chappell ${ }^{3}$, Jérémy Dargent ${ }^{4}$ (D) Stephen A. Fuselier ${ }^{5,6}$ (D), Stein Haaland ${ }^{7,8}$ (D), \\ Benoit Lavraud ${ }^{9}$ (D), Wenya Li $^{10}$, Paul Tenfjord ${ }^{8}$ (D), Sergio Toledo-Redondo9 \\ and Sarah K. Vines ${ }^{11}$ (iD \\ ${ }^{1}$ Swedish Institute of Space Physics, Uppsala, Sweden, ${ }^{2}$ KTH Royal Institute of Technology, Stockholm, Sweden, \\ ${ }^{3}$ Vanderbilt Dyer Observatory, Department of Physics and Astronomy, Vanderbilt University, Nashville, TN, USA, \\ ${ }^{4}$ Physics Department “Enrico Fermi”, University of Pisa, Pisa, Italy, ${ }^{5}$ Southwest Research Institute, San Antonio, TX, \\ USA, ${ }^{6}$ Department of Physics and Astronomy, University of Texas at San Antonio, San Antonio, TX, USA, ${ }^{7}$ Max-Planck \\ Institute for Solar Systems Research, Göttingen, Germany, ${ }^{8}$ Birkeland Centre for Space Science, University of Bergen, \\ Bergen, Norway, ${ }^{9}$ Institut de Recherche en Astrophysique et Planétologie, Université de Toulouse, CNRS, UPS, CNES, \\ Toulouse, France, ${ }^{10}$ State Key Laboratory of Space Weather, National Space Science Center, Chinese Academy of \\ Sciences, Beijing, China, ${ }^{11}$ The Johns Hopkins University Applied Physics Laboratory, Laurel, MD, USA
}

Abstract We present Magnetospheric Multiscale mission (MMS) observations of Hall physics in the magnetotail, which compared to dayside Hall physics is a relatively unexplored topic. The plasma consists of electrons, moderately cold ions $(T \sim 1.5 \mathrm{keV})$ and hot ions $(T \sim 20 \mathrm{keV})$. MMS can differentiate between the cold ion demagnetization region and hot ion demagnetization regions, which suggests that MMS was observing multiscale Hall physics. The observed Hall electric field is compared with a generalized Ohm's law, accounting for multiple ion populations. The cold ion population, despite its relatively high initial temperature, has a significant impact on the Hall electric field. These results show that multiscale Hall physics is relevant over a much larger temperature range than previously observed and is relevant for the whole magnetosphere as well as for other astrophysical plasma.

\section{Introduction}

In magnetic reconnection, reconfiguration of the magnetic topology and particle demagnetization, breaking the frozen-in condition $\mathbf{E}+\mathbf{v} \times \mathbf{B}=0$, allows for mixing of plasma across boundaries and energy transfer (Vasyliunas, 1975). Particle demagnetization occurs inside magnetic structures which are thinner than the particle's gyro radius (Büchner \& Zelenyi, 1989). Therefore, particle demagnetization depends on the particle's mass, energy, and the magnetic field. Near the center of a thin current sheet, a particle is expected to demagnetize and assume a meandering orbit, when its gyro radius exceeds the distance to the center of the current sheet, the so called meandering orbit scale (Ishizawa \& Horiuchi, 2005; Kuznetsova et al., 1998).

Hall physics can arise anywhere ion demagnetization occurs. The differential motion between magnetized electrons and demagnetized ions generates the Hall current, which sets up the Hall electric and magnetic fields (Nagai et al., 2001; Sonnerup, 1979). Magnetic reconnection is often considered a two-scale process due to the large difference between the scales on which electrons and ions become demagnetized. The two-scale model of reconnection assumes a single electron population and a single ion population. Observation and simulations of reconnection with ion populations with different temperatures have shown that there exists an outer hot ion demagnetization region with reduced Hall current and an inner cold ion demagnetization region (André et al., 2010; André et al., 2016; Divin et al., 2016; Toledo-Redondo et al., 2015). The Hall current introduces instabilities which may trigger reconnection onset, which means that multiscale Hall physics, with reduced Hall current, may change the micro physics of magnetic reconnection (Biskamp et al., 1997; Birn et al., 2001; Shay et al., 2001). However, 2-D particle-in-cell simulations have shown that introducing cold ions, while keeping the density constant, does not significantly change reconnection rate (Dargent et al., 2017; Divin et al., 2016).

Many observations of multiscale Hall physics come from the dayside magnetopause, and may not be applicable in the magnetotail, where the conditions are different. Dayside reconnection has asymmetric inflow 
conditions, with low magnetospheric density and high magnetosheath density. This causes the Hall fields to become more pronounced on the magnetospheric side, so that the Hall electric field becomes almost monopolar (Mozer et al., 2008; Pritchett \& Mozer, 2009). Furthermore, at the dayside magnetopause, cold ions are mainly found on the magnetospheric side, introducing a cold ion asymmetry, whereas in the magnetotail cold ions can be present on both sides of the neutral sheet.

The lower density in the magnetotail makes it more difficult to produce accurate particle distributions. In addition, the Hall electric field in the magnetotail is often close to $Z_{\mathrm{GSE}}$, which for many missions (i.e., Cluster; Time History of Events and Macroscale Interactions during Substorms; and Magnetospheric Multiscale mission, MMS) is close to the satellite's spin axis. This means that the accuracy of the measurements of the Hall electric field is largely dependent on the capabilities of the axial electric double probes, or in the case of Cluster, the lack thereof.

It is known that in many cases the cold $(T<60 \mathrm{eV})$ ions dominate the tail lobe plasma (André \& Cully, 2012; André et al., 2015; Engwall et al., 2008, 2009; Huddleston et al., 2005). During high geomagnetic activity, increased ionospheric ion outflow and enhanced convection cause an increased transport of cold ions to the plasma sheet (Li et al., 2013). It has been unclear whether cold (tens of electronvolts) lobe ions that enter the hot $(>10 \mathrm{keV})$ dense plasma sheet can remain cold enough to behave as a separate population.

In a recent paper, Alm et al. (2018) used 3-D electric field measurements and high time-resolution particle distributions from the MMS to characterize the Hall electric field during a partial plasma sheet crossing. A majority of the Hall electric field was supported by the $\mathbf{j} \times \mathbf{B}$ term of the generalized Ohm's law, with significant contributions from the cold ion convective electric field and divergence of the electron pressure (Alm et al., 2018). In the aforementioned paper, the plasma sheet crossing was superficial, the reconnecting component $\left(B_{X}\right)$ was always $<7 \mathrm{nT}$, and the satellites never crossed the neutral sheet. Furthermore, the cold ions consisted of lobe ions $(T<100 \mathrm{eV})$ which had entered the plasma sheet without prior heating, resulting in an extreme temperature difference between the hot and cold ions.

In this paper we present a deep partial plasma sheet crossing $\left(\min \left(B_{X}\right)=0.6 \mathrm{nT}\right)$, allowing us to study the evolution of the cold ions down to the central plasma sheet. Many previous studies of multiscale Hall physics have focused on events where the cold ions are up to 2 orders of magnitude colder than the plasma sheet ions. In this event MMS observes mixing of hot $(T \sim 20 \mathrm{keV})$ plasma sheet ions and preheated, moderately cold $(T \sim 1.5 \mathrm{keV})$ lobe ions. This allows us to investigate if previous findings are applicable when the temperature difference between the two ion populations is less extreme, which would suggest that multiscale Hall physics is relevant over a larger temperature range than previously thought.

\section{Observations}

MMS measures electric and magnetic fields using two fluxgate magnetometers (FGM), axial and spin plane electric double probes (EDP), a search coil magnetometer, and an electron drift instrument (Ergun et al., 2016; Le Contel et al., 2016; Lindqvist et al., 2016; Russell et al., 2016; Torbert, Russell, et al., 2016; Torbert, Vaith, et al., 2016). The fast plasma investigation (FPI) provides 3-D electron and ion distributions in the energy range $10 \mathrm{eV}$ to $30 \mathrm{keV}$, with a burst-mode time resolution of 30 and $150 \mathrm{~ms}$, respectively (Pollock et al., 2016). The hot plasma composition analyzer (HPCA) provides energy, angular distribution, and composition of ions $1 \mathrm{eV}$ to $40 \mathrm{keV}$ and is complemented by the energetic ion spectrometer (EIS), which covers 20- to >500-keV protons (Mauk et al., 2016; Young et al., 2016).

The event described here occurred on 21 August 2017, around 17:57 UT. MMS was located in the near-Earth tail, at $[-11.7,7.3,6.9] R_{E}$ (GSM), just north of the nominal plasma sheet boundary. While Hall physics is usually associated with the more distant tail, Hall current systems have been reported in the near-Earth tail, for example, Nakamura et al. (2018). The mean satellite separation was $20 \mathrm{~km}$, minimum separation of $17 \mathrm{~km}$ between MMS3 and MMS4, and maximum separation of $29 \mathrm{~km}$ between MMS2 and MMS4.

Figure 1 is an overview of the event as observed by MMS2. MMS2 was initially located in the plasma sheet boundary layer, close to the plasma sheet. This is inferred from the strong magnetic fields (Figure 1a), the lack of high-energy ions (Figures $1 \mathrm{~b}$ and 1c) and intermittent observation of $\sim 3-\mathrm{keV}$ plasma sheet electrons (Figure 1e). The ion temperature was $\sim 1.5 \mathrm{keV}$, more than 1 order of magnitude higher than typically observed in the lobes but an order of magnitude lower than the $\sim 20 \mathrm{keV}$ later observed inside the central plasma sheet. 

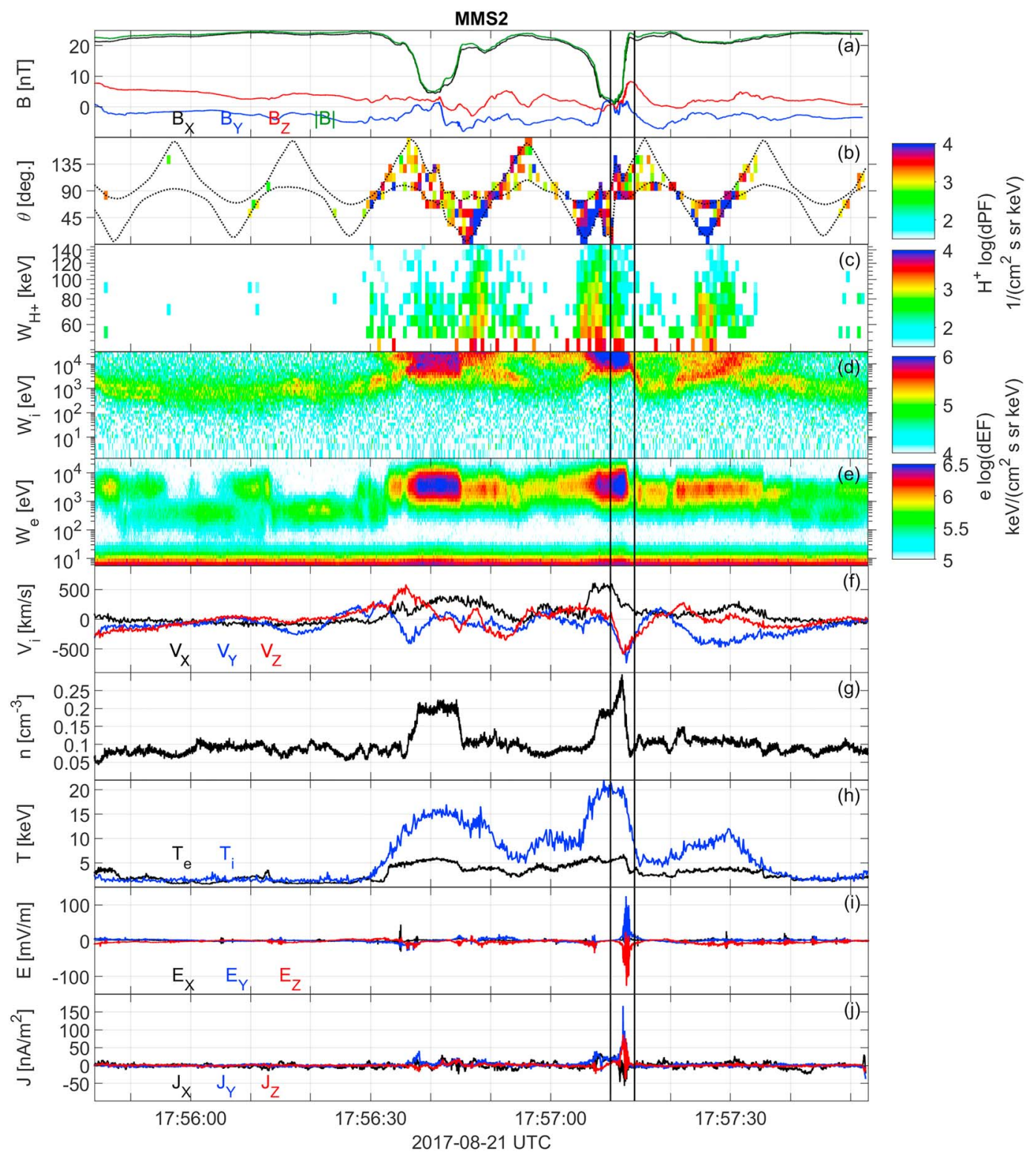

Figure 1. Overview of the event as observed by MMS2, vector quantities in GSM. (a) Magnetic field (FGM), (b) hydrogen ion pitch angle spectrogram (EIS), (c) hydrogen ion energy spectrogram (EIS). The dashed lines denote the field of view of EIS. (d) Ion energy spectrogram (FPI), (e) electron energy spectrogram (FPI), (f) ion bulk velocity (FPI), (g) plasma density (FPI), (h) plasma temperature (FPI), (i) electric field (EDP), (j) curlometer current (FGM). MMS = Magnetospheric Multiscale mission; FGM = fluxgate magnetometers; $\mathrm{EDP}=$ electric double probes; FPI $=$ fast plasma investigation; EIS = energetic ion spectrometer.

Between 17:56:30 UT and 17:57:20 UT, MMS observed two plasma sheet crossings, indicated by the increase in plasma temperature and density, and the decrease in magnetic field strength. The lack of a $B_{X}$ reversal indicates that both plasma sheet crossings were partial. The two plasma sheet crossings were associated with increased ion fluxes at energies $>1 \mathrm{keV}$, observed by FPI and EIS. The 20-s periodicity in the EIS particle flux is from the time required for EIS to achieve full sky coverage by sweeping the sky over the course of one satellite spin period.

The second partial plasma sheet crossing exhibited a deep magnetic minimum $(|B|<1 \mathrm{nT})$ and an ion temperature of more than $20 \mathrm{keV}$ (Figure 1h), suggesting MMS was close to the neutral sheet. The inbound leg did not exhibit strong electric fields or currents but an enhanced earthward ion flow of $\sim 500 \mathrm{~km} / \mathrm{s}$. The outbound leg exhibited a negative $E_{Z}$ of more than $100 \mathrm{mV} / \mathrm{m}$, which corresponds to the expected direction 
of the Hall electric field in the northern hemisphere, and currents in excess of $140 \mathrm{nA} / \mathrm{m}^{2}$. Furthermore, MMS observed strong flows toward the neutral sheet, consistent with the inflow direction as well as cross tail flows.

Figure 2 focuses on the outbound leg of the second plasma sheet crossing. The orientation of the plasma sheet was determined using minimum variance analysis of the magnetic field (Sonnerup \& Cahill Jr, 1967). The minimum variance analysis of the magnetic field shows that for both the inbound and outbound pass, $L$ is $\sim X_{\mathrm{GSM}}\left[\begin{array}{lll}0.95-0.10 & 0.31\end{array}\right], M$ is [ $\left.-0.180 .620 .77\right]$, and $N$ is [ $\left.-0.27-0.780 .56\right]$. These results are also supported by maximum variance analysis of the current and maximum directional derivative analysis of the magnetic field (Haaland et al., 2004; Shi et al., 2005). From here on, LMN coordinates will be used to describe vector quantities.

The agreement between the electric field and the electron convective electric field $-\mathbf{v}_{\mathbf{e}} \times \mathbf{B}$, seen in Figures $2 \mathrm{~b}-2 \mathrm{~d}$, indicates that the electrons are magnetized. In contrast, $-\mathbf{v}_{\mathbf{i}} \times \mathbf{B}$ is smaller than the observed electric field but nonzero, indicating that the ions are demagnetized. The presence of both demagnetized ions and magnetized electrons indicates that MMS was located inside a Hall region. Since $-\mathbf{v}_{\mathbf{i}} \times \mathbf{B}$ was calculated using the FPI bulk ion moments, which do not cover the full energy range of the hot ion population. HPCA spans a higher energy range, covering more of plasma sheet ion, but the time resolution (10 s) is insufficient for studying Hall physics. As seen in Figure 1b, EIS detects higher ion fluxes for low pitch angles, which indicates they are mostly field aligned and should not contribute to $-\mathbf{v}_{\mathbf{i}} \times \mathbf{B}$.

As seen in Figure 2e, inside the plasma sheet it is hard to distinguish between the two ion populations based on their energy. The two populations have comparable total energy but the cold ions have more energy in the bulk velocity than thermal velocity. Figure $2 \mathrm{f}$, illustrates the difference between the two ion populations. Outside the central plasma sheet (after 17:57:13 UT), the ion flux is enhanced for pitch angles around $90^{\circ}$, which is expected from magnetized particles with comparable bulk and thermal velocity. Deeper inside the plasma sheet, MMS observes moderately cold ions around $90^{\circ}$ pitch angle and hotter ions at pitch angles $<45^{\circ}$. The moderately cold ions will hereafter be referred to as cold ions. The cold ion moments were calculated by restricting the integration of the distribution function to pitch angles between $45^{\circ}$ and $135^{\circ}$, seen in Figure $2 f$.

Between 17:57:12.4 and 17:57:12.8 UT the electron energy spectrograms (Figure 2g and 2h) exhibit periodical modulations in the electron flux, coinciding with oscillations observed in the magnetic field (Figure 2a), electric field (Figures 2b-2d), and current (Figure 2i). These oscillations at the lower-hybrid frequency $(\sim 15 \mathrm{~Hz})$ cannot be resolved by the FPI ion time resolution $(150 \mathrm{~ms})$. The waves propagate along the $-M$ direction, which is similar to lower-hybrid waves driven by interaction between cold and hot ions that have been observed at the magnetopause (Graham et al., 2017), suggested as a mechanism for heating cold ions (Toledo-Redondo et al., 2017). Around 17:57:12.1 UT MMS observed a $j_{M}$ current sheet with a peak current of $180 \mathrm{nA} / \mathrm{m}^{2}$ and a thickness of $\sim 24 \mathrm{~km}\left(\sim 1.8 \rho_{\text {ce }}\right)$, denoted by the first vertical line in Figure 2 . At approximately 17:57:12.2 UT, MMS observed weaker but thinner current sheet, with a thickness $\sim 11 \mathrm{~km}\left(\sim 0.8 \rho_{\text {ce }}\right)$, denoted by the second vertical line.

The motion of MMS, normal to the plasma sheet, was determined using the spatiotemporal difference method on the $B_{L}$ component (Denton et al., 2016; Shi et al., 2005). Prior to 17:57:12 UT, where $B_{L}<5 \mathrm{nT}$, the magnetic field and its gradients are too weak to provide an accurate estimate of the motion of MMS. The extended region of low and constant $B_{L}$, suggests that MMS is mostly moving in the plane parallel to the neutral sheet. After 17:57:12 UT, the plasma sheet can be modeled as a Harris sheet, by fitting $B_{L}$ as a function of the position along the normal direction (Figure $2 \mathrm{j}$ ). The meandering orbit scale $\left(l_{m}\right)$, on which particles become demagnetized, is defined as where the distance to the neutral line is equal to the local gyro radius, $\rho_{c}\left(l_{m}\right)=l_{m}$ (Ishizawa \& Horiuchi, 2005; Kuznetsova et al., 1998). Using our Harris sheet model and cold ion gyro radius, calculated using the local magnetic field and cold ion temperature, we can estimate that the cold ion meandering orbit scale was $318 \mathrm{~km}$.

HPCA shows that heavier ions were present but accounted for $<1 \%$ of the total number density (not shown). Therefore, the Hall electric field was modeled using one electron population, a cold hydrogen ion population, and one hot hydrogen ion population. The electric field $\mathbf{E}$ can be expressed as

$$
\mathbf{E}=\frac{1}{e n} \mathbf{j} \times \mathbf{B}-\frac{n_{i c}}{n} \mathbf{v}_{\mathbf{i c}} \times \mathbf{B}-\frac{n_{i h}}{n} \mathbf{v}_{\mathbf{i h}} \times \mathbf{B}-\frac{1}{e n} \nabla \cdot \mathbf{P}_{\mathbf{e}}
$$



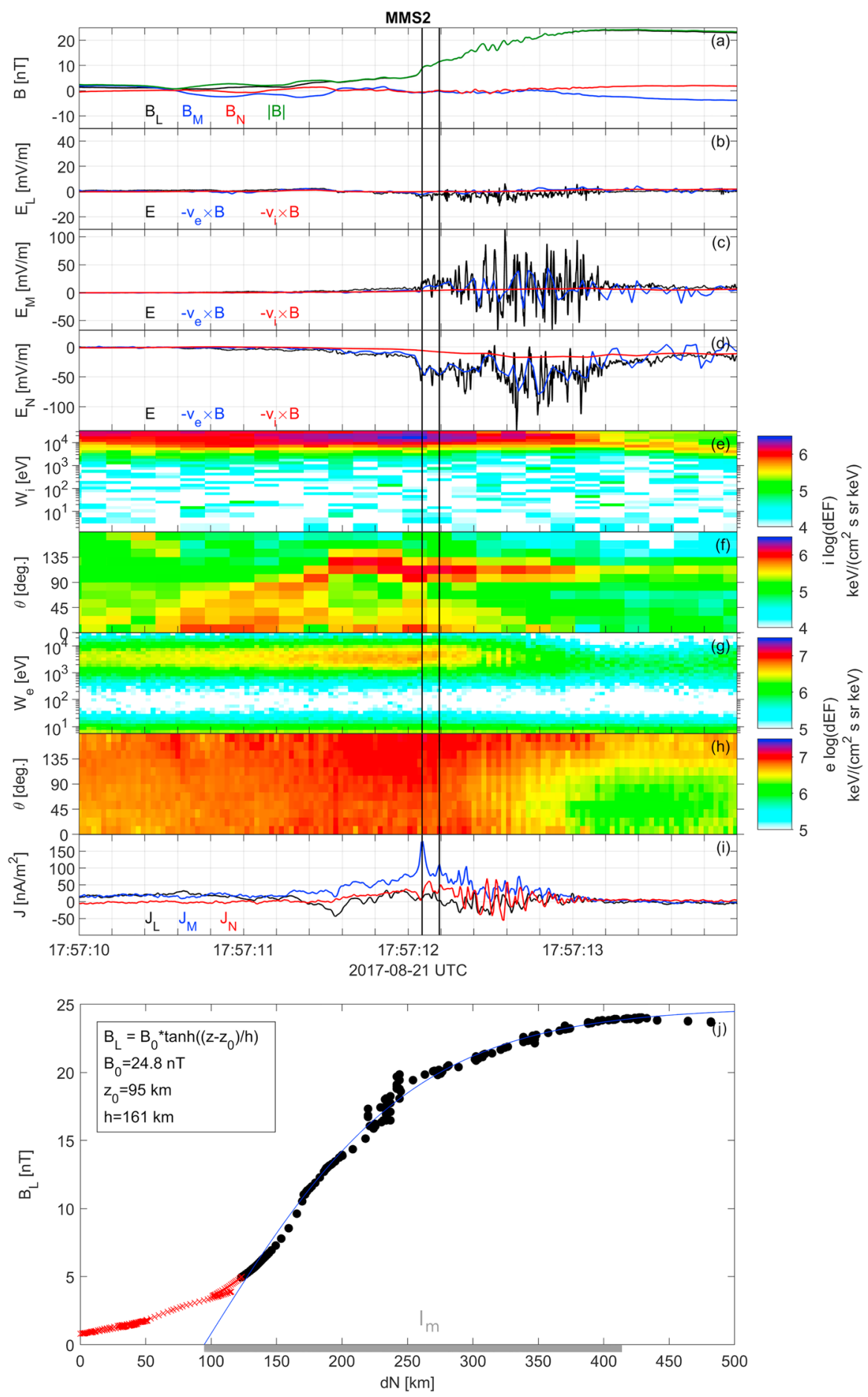

Figure 2. Electron and ion spectrograms from MMS3. (a) Magnetic field (FGM); (b)-(d) electric field from EDP (black), $-\mathbf{v}_{\mathbf{e}} \times \mathbf{B}$ (blue), and $-\mathbf{v}_{\mathbf{i}} \times \mathbf{B}$ (red); (e) ion energy spectrogram (FPI); (f) ion pitch angle spectrogram (FPI); (g) electron energy spectrogram (FPI); (h) electron pitch angle spectrogram (FPI); (i) curlometer current (FGM); (j) $B_{L}$ as a function of position along $\mathrm{N}$. The blue line represent a Harris type current sheet fitted to the data. The gray bar along the $x$ axis represents the meandering orbit scale or the cold ions. The vertical lines in panels (a)-(i) denote the position of the two thin current sheets. MMS = Magnetospheric Multiscale mission; FGM = fluxgate magnetometers; $\mathrm{EDP}=$ electric double probes; $\mathrm{FPI}=$ fast plasma investigation. 
where $e$ is the elemental charge, $n$ is the plasma density, $\mathbf{j}$ is the current (Robert et al., 1998), $\mathbf{B}$ is the magnetic field, $n_{\mathrm{ic}}\left(n_{\mathrm{ih}}\right)$ is the cold(hot) ion density, $\mathbf{v}_{\mathrm{ic}}\left(\mathbf{v}_{\mathrm{ih}}\right)$ is the bulk velocity of the cold(hot) ions, and $\mathbf{P}_{\mathbf{e}}$ is the electron pressure tensor (Stiles et al., 1978). Electron inertia is ignored since we are focusing on ion scale physics, where electron inertia plays little role (André et al., 2016; Toledo-Redondo et al., 2015).

The cold ion moments were calculated by restricting the integration of the distribution function to pitch angles between $45^{\circ}$ and $135^{\circ}$, discussed previously. The remaining ions were considered to be part of the hot ion population. Note that part of the hot ion population extends above the FPI energy range. However, as seen in Figure $1 \mathrm{~b}$, these ions are mostly field aligned and should not contribute significantly to $\mathbf{v}_{\text {ih }} \times \mathbf{B}$. The hot ion density can be estimated by assuming that $n_{e}=n_{i}=n_{\mathrm{ic}}+n_{\mathrm{ih}}$. As discussed by Alm et al. (2018), the hot ions are accounted for indirectly, as part of the Hall current, which is calculated using the curlometer method rather than from particle moments.

The terms of the right-hand side (RHS) of equation (1) represent three characteristic length scales. The $1 /(e n) \mathbf{j} \times \mathbf{B}$ and $\left(n_{\mathrm{ih}} / n\right) \mathbf{v}_{\mathrm{ih}} \times \mathbf{B}$ terms can be used to identify the outer edge of the hot ion demagnetization region. Using the $1 /(e n) \mathbf{j} \times \mathbf{B}$ term is preferable over using the $-v_{i h} \times \mathbf{B}$ term since MMS cannot observe the entire hot ion population with high enough time resolution. Since the $\mathbf{j} \times \mathbf{B}$ term arises from the differential motion between magnetized and demagnetized particles, its first appearance suggest that the hottest ions are demagnetized. The cold ion term $\left(-\left(n_{\text {ic }} / n\right) \mathbf{v}_{\mathbf{i c}} \times \mathbf{B}\right)$ describes the behavior of the cold ions, where $\mathbf{E} \neq-\mathbf{v}_{\mathbf{i c}} \times \mathbf{B}$ indicates a cold ion demagnetization region. Lastly, a finite electron pressure term $\left(1 /(e n) \nabla \cdot \mathbf{P}_{\mathbf{e}}\right)$ is indicative of being inside an electron demagnetization region.

Figure $3 \mathrm{~b}$ compares the observed electric field with the electron, cold ion, and hot ion convective electric fields. Between 17:57:11.5 UT and 17:57:13.2 UT, $-\mathbf{v}_{\mathbf{i c}} \times \mathbf{B}$ is finite but smaller than the observed electric field. This difference cannot be explained by the lower time resolution of the FPI ion moments. After 17:57:13.2 UT, the electric field and cold ion convective electric field are in agreement, indicating that the cold ions are frozen in. The hot ion convective electric field $\left(-\mathbf{v}_{\mathrm{ih}} \times \mathbf{B}\right)$ is always $<4 \mathrm{mV} / \mathrm{m}$ and does not track the evolution of the observed electric field, suggesting that the hot ions are demagnetized. The agreement between $-\mathbf{v}_{\mathbf{e}} \times \mathbf{B}$ and the observed electric field indicates that on the FPI time scale, $30 \mathrm{~ms}$, the electrons were magnetized. The smoother appearance of the electrons, compared to the electric field, is primarily due to the lower sample rate of FPI compared to EDP. The oscillations in the electron convective electric field seen after 17:57:13.2 UT are primarily due to the low counts.

Figure $3 \mathrm{c}$ shows the normal component of $\mathbf{j} \times \mathbf{B}$, using the full 7.8-ms time resolution of FGM. Since the curlometer current is both a spatial and a temporal average, it underestimates current sheets which have thickness smaller than, or comparable to, the $20-\mathrm{km}$ spacecraft separation. The normal component of the $\mathbf{j} \times \mathbf{B}$ term is primarily generated by the out-of-plane $\left(j_{M}\right)$ current. For a single spacecraft, performing a normal crossing of a 1-D current sheet, $j_{M}$ can be estimated by $\mu_{0} j_{M}=\Delta B_{L} / \Delta N$, where $\Delta N$ is the thickness along the $N$ direction, given by timing analysis. The blue circles in Figure $3 \mathrm{c}$ show $\mathbf{j} \times \mathbf{B}$ estimated using the single spacecraft method, performed on each satellite individually, and averaged over all satellites. The current sheets observed between 17:57:12.0 and 17:57:12.2 UT have a thickness of $\sim 24 \mathrm{~km}\left(\sim 1.8 \rho_{\text {ce }}\right)$ and $\sim 11 \mathrm{~km}$ $\left(\sim 0.8 \rho_{\text {ce }}\right)$, respectively, and the curlometer method underestimates the peak current by a factor $\sim 1.2$ and $\sim 1.5$ compared to the single spacecraft method. For the two current sheets observed between 17:57:12.3 and 17:57:12.4 UT, the curlometer and single-spacecraft method agree within 5\%. This is expected since the current sheets have a thickness $34 \mathrm{~km}\left(\sim 3 \rho_{\text {ce }}\right)$ and $41 \mathrm{~km}\left(\sim 4 \rho_{\text {ce }}\right)$, which is larger than the spacecraft separation. After 17:57:12.4 UT, the single-spacecraft method cannot be used since the lower-hybrid dominated current sheets cannot be described using our simple 1-D approximation.

Figure $3 \mathrm{~d}$ shows the normal component of the divergence of the electron pressure, which is calculated across the tetrahedron. This makes it sensitive to artifacts introduced when resampling and comparing the data from different satellites. This is especially true for temporal variations which cannot be resolved by FPI, as is the case for the lower-hybrid waves observed between 17:57:12.4 and 17:57:12.8 UT (not shown). Temporal variations which exhibits a phase difference across MMS can then be interpreted as spatial gradients. The only feature which stands out is the peak observed at approximately 17:57:12.2 UT, with a maximum of $1 \mathrm{pPa} / \mathrm{km}$. This feature coincides with the second thin current sheet, discussed above.

As seen in Figure 3e, the plasma sheet density is $\sim 0.19 \mathrm{~cm}^{-3}$ with $\sim 40 \%$ cold ions and $\sim 60 \%$ hot ions. As MMS moves toward the plasma sheet boundary layer the plasma density increases initially, reaching a peak 


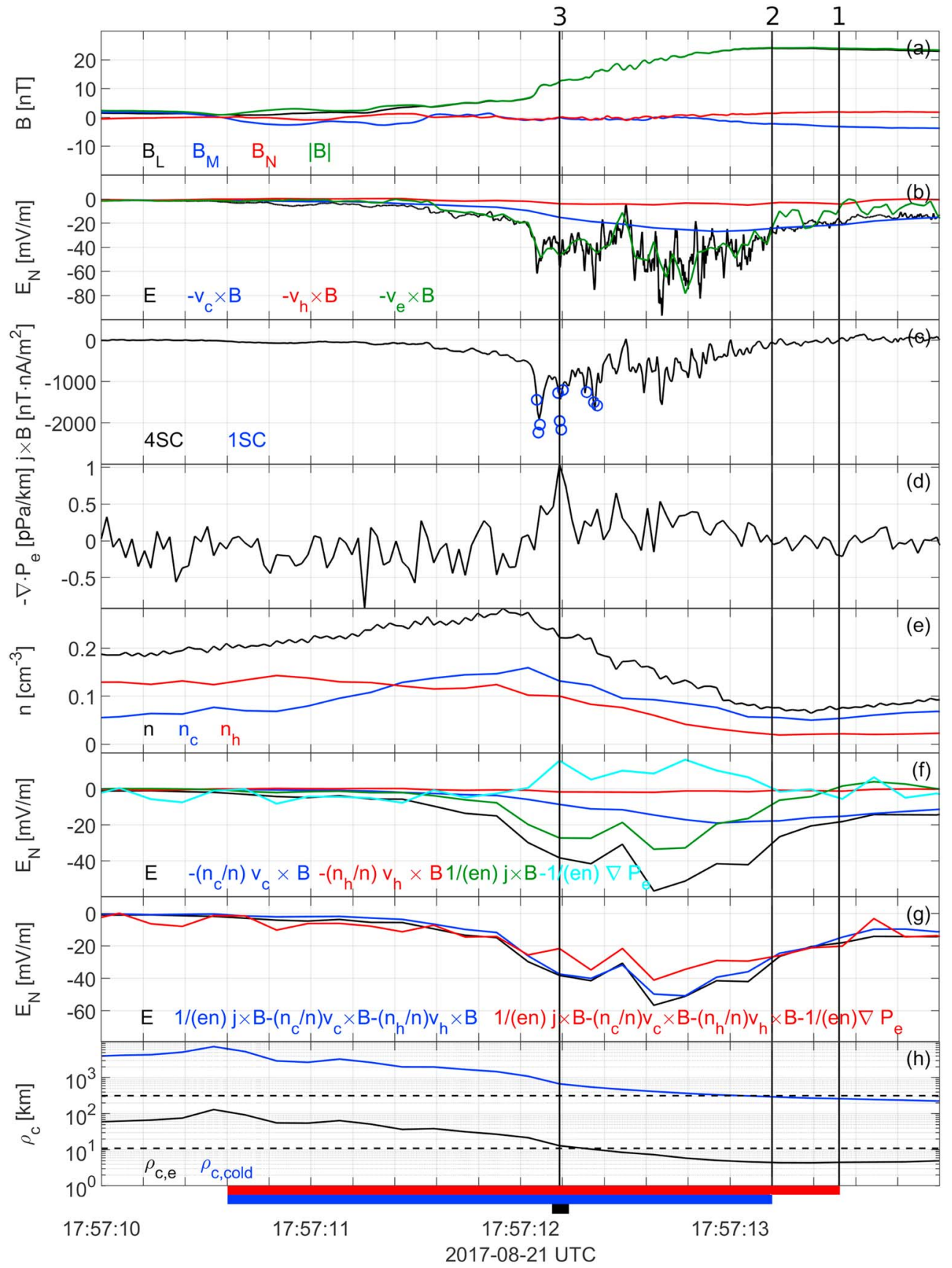

Figure 3. Normal component of generalized Ohm's law. (a) Magnetic field; (b) electric field from EDP (black), $-v_{e} \times \mathbf{B}$ (blue), and $-v_{i} \times \mathbf{B}$ (red); (c) normal component of $\mathbf{j} \times \mathbf{B}$, calculated from curlometer (black) and single-spacecraft method (blue); (d) divergence of the electron pressure; (e) plasma density (black), cold ion density (blue), andhot ion density (red); (f) terms of the generalized Ohm's law; (g) electric field and sum of the right-hand side of generalized Ohm's law; and (h) gyro radii of electrons (black) and cold ions (blue). The upper horizontal dashed line indicates the cold ion meandering orbit scale $l_{m}$ and the lower dashed line indicates full-width half-maximum of the current sheet observed at 17:57:12.2 UT. The bars along the time axis indicate the electron demagnetization region (black), cold ion demagnetization region (blue), and hot ion demagnetization region (red). EDP $=$ electric double probes. 
density of $\sim 0.27 \mathrm{~cm}^{-3}$ at 17:57:12 UT. This is due to increasing cold ion density, while the hot ion density is decreasing.

Figure $3 \mathrm{f}$ compares the terms of equation (1). The data have been downsampled to the time resolution of the FPI ion moments (150 ms). After 17:57:13 UT, the cold ion term dominates, with a peak magnitude of $21 \mathrm{mV} / \mathrm{m}$. The magnitude of the hot ion term never exceeds $4 \mathrm{mV} / \mathrm{m}$. As can be inferred from Figures $3 \mathrm{~b}$ and $3 \mathrm{e}$ this is not due to an absence of hot ions but rather that $-v_{i h} \times \mathbf{B}$ is small, suggesting that hot ions are present but demagnetized. Prior to 17:15:13 UT the $\mathbf{j} \times \mathbf{B}$ term dominates the RHS of equation (1).

The $\mathbf{j} \times \mathbf{B}$ term exhibits the same trend as the electric field but can only account for $\sim 50 \%$ of the observed electric field. The $\mathbf{j} \times \mathbf{B}$ term exhibits the largest peak value of the four terms $(-33 \mathrm{mV} / \mathrm{m})$, similar to previous studies of dayside (André et al., 2004; Khotyaintsev et al., 2006; Retinò et al., 2006) and magnetotail Hall fields (Alm et al., 2018).

For most of the event, the electron pressure term has an average value of $\sim 0$, with considerable noise. Between 17:57:12 UT and 17:57:13 UT, MMS observed a finite electron pressure term, with two peaks of $\sim 16 \mathrm{mV} / \mathrm{m}$. The first peak corresponds to when MMS observed the two thin current sheets and the peak in $-\nabla \cdot \mathbf{P}_{\mathbf{e}}$ (Figure $3 \mathrm{~d}$ ). The second peak in the electron pressure term of Figure $3 \mathrm{f}$ does not correspond to any feature in Figure 3d and appears to mainly be generated from noise which is amplified by the low plasma density through the 1/en scaling factor in equation (1).

As can be seen in Figure $3 g$, the observed electric field can be accurately described by the sum of the $\mathbf{j} \times \mathbf{B}$, $\mathbf{v}_{\mathbf{i c}} \times \mathbf{B}$ and $\mathbf{v}_{\mathbf{i h}} \times \mathbf{B}$ terms of equation (1). This combination tends to underestimate the electric field by $\sim 7 \%$. Including the $\nabla \cdot \mathbf{P}_{\mathbf{e}}$ term does not provide a better estimate of the electric field and underestimates the electric field by $\sim 25 \%$ on average.

\section{Discussion and Conclusions}

We argue that MMS encountered a hot ion demagnetization region, a cold ion demagnetization region, and an electron demagnetization region. The extent of the three demagnetization regions are indicated by the vertical lines and the colored bars along the time axis of Figure 3. MMS encountered the outer edge of the hot ion demagnetization region at 17:57:13.5 UT, denoted by the vertical line labeled 1. After this time MMS does not observe a significant $\mathbf{j} \times \mathbf{B}$ term, indicating that all particle populations are magnetized.

The outer edge of the cold ion demagnetization region was observed at 17:57:13.2 UT, denoted by the vertical line labeled 2. This is indicated by the transition from $\mathbf{E} \neq-v_{i c} \times \mathbf{B}$ to $\mathbf{E}=-\mathbf{v}_{\text {ic }} \times \mathbf{B}$. This happens where the local gyro radius of the cold ions exceeds the meandering orbit scale, which is expected from theory (Ishizawa \& Horiuchi, 2005; Kuznetsova et al., 1998). The small separation between the hot and cold ion demagnetization regions can at a first glance appear problematic. In this particular case, the cold ions are not lobes ions, with a temperature of a few tens of electronvolts, but have been heated to $\sim 1.5 \mathrm{keV}$ inside the plasma sheet boundary layer. However, the temperature is still significantly lower than in the central plasma sheet ( $20 \mathrm{keV})$ and cold enough to warrant treatment as a separate ion population.

We believe that MMS briefly crossed an electron demagnetization region, inside the thin current sheet observed at 17:57:12.2 UT, denoted by the vertical line labeled 3. The current sheet's thickness was $11 \mathrm{~km}$, $\sim 0.8 \rho_{\text {ce }}$, which suggests it is a possible site for electron demagnetization. This would explain the peak in $-\nabla \cdot \mathbf{P}_{\mathbf{e}}$ observed at the same time (Figure 3d). Unfortunately, the combination of FPI not being able to resolve this structure and the highly variable electric field makes it impossible to unambiguously determine if electrons were in fact demagnetized by comparing the electric field with $-\mathbf{v}_{\mathbf{e}} \times \mathbf{B}$.

If MMS did briefly cross an electron demagnetization region, why does the agreement between the observed electric field and the RHS of equation (1) become worse when including the electron pressure term? As shown in Figure $3 \mathrm{c}$ this current sheet cannot be resolved by the curlometer method and we underestimate the $\mathbf{j} \times \mathbf{B}$ term by a factor $\sim 1$.5. Inside this current sheet, the RHS of equation (1), including the electron pressure term and using the single spacecraft method to calculate the $\mathbf{j} \times \mathbf{B}$ term, differs from the observed electric field by $\sim 5 \%$. Thus, inside the thin current sheet, the electron pressure term is required to explain the observed electric field suggesting that MMS observed local electron demagnetization. The thin current sheet is only barely resolved when using the 7.8-ms resolution of FGM and cannot be resolved with the 30-ms 
Acknowledgments

We acknowledge support by the Swedish National Space Agency contracts SNSB 176/15 and 158/16 Work at IRAP was supported by the Université de Toulouse, CNRS, and CNES. This study was facilitated by the International Space Science Institute (ISSI) through the research team studying cold plasma of ionospheric origin. This paper was made possible by the hard work of the MMS Team. All data used in this paper are publicly available at MMS Science Data Center (https://lasp.colorado.edu/mms/sdc/ public/). electron distributions, nor the 150-ms ion distributions. This means that it does not have a significant impact on the terms of equation (1), which are calculated using the time resolution of the FPI ion distributions.

To summarize, compared to many previous studies of cold ions, the initial temperature of the cold ion population is relatively high, $\sim 1.5 \mathrm{keV}$, more than an order of magnitude higher than those of the typical cold lobe ions. Despite this, the cold ion term of equation 1 is finite except in the central plasma sheet. This indicates that the multiscale physics introduced by cold ions is relevant over a wider range of plasma temperatures than has been discussed in previous studies and thus relevant for the whole magnetosphere as well as other astrophysical plasma. These findings further highlight the importance of considering a multipopulation/multiscale perspective when modeling Hall physics and magnetic reconnection.

\section{References}

Alm, L., André, M., Vaivads, A., Khotyaintsev, Y. V., Torbert, R. B., Burch, J. L., \& Mauk, B. H. (2018). Magnetotail Hall physics in the presence of cold ions. Geophysical Research Letters, 45, 10,941-10,950. https://doi.org/10.1029/2018GL079857

André, M., \& Cully, C. M. (2012). Low-energy ions: A previously hidden solar system particle population. Geophysical Research Letters, 39, L03101. https://doi.org/10.1029/2011GL050242

André, M., Li, K., \& Eriksson, A. I. (2015). Outflow of low-energy ions and the solar cycle. Journal of Geophysical Research: Space Physics, 120, 1072-1085. https://doi.org/10.1002/2014JA020714

André, M., Li, W., Toledo-Redondo, S., Khotyaintsev, Y. V., Vaivads, A., Graham, D. B., \& Saito, Y. (2016). Magnetic reconnection and modification of the Hall physics due to cold ions at the magnetopause. Geophysical Research Letters, 43, 6705-6712. https://doi.org/10. 1002/2016GL069665

André, M., Vaivads, A., Buchert, S. C., Fazakerley, A. N., \& Lahiff, A. (2004). Thin electron-scale layers at the magnetopause. Geophysical Research Letters, 31, L03803. https://doi.org/10.1029/2003GL018137

André, M., Vaivads, A., Khotyaintsev, Y. V., Laitinen, T., Nilsson, H., Stenberg, G., \& Trotignon, J. G. (2010). Magnetic reconnection and cold plasma at the magnetopause. Geophysical Research Letters, 37, L22108. https://doi.org/10.1029/2010GL044611

Birn, J., Drake, J., Shay, M., Rogers, B., Denton, R., Hesse, M., et al. (2001). Geospace Environmental Modeling (GEM) magnetic reconnection challenge. Journal of Geophysical Research, 106(A3), 3715-3719.

Biskamp, D., Schwarz, E., \& Drake, J. F. (1997). Two-fluid theory of collisionless magnetic reconnection. Physics of Plasmas, 4(4), 1002-1009. https://doi.org/10.1063/1.872211

Büchner, J., \& Zelenyi, L. M. (1989). Regular and chaotic charged particle motion in magnetotaillike field reversals: 1 . Basic theory of trapped motion. Journal of Geophysical Research, 94(A9), 11,821-11,842.

Dargent, J., Aunai, N., Lavraud, B., Toledo-Redondo, S., Shay, M. A., Cassak, P. A., \& Malakit, K. (2017). Kinetic simulation of asymmetric magnetic reconnection with cold ions. Journal of Geophysical Research: Space Physics, 122, 5290-5306. https://doi.org/10.1002/ 2016JA023831

Denton, R. E., Sonnerup, B. U., Hasegawa, H., Phan, T. D., Russell, C. T., Strangeway, R. J., \& Torbert, R. B. (2016). Motion of the MMS spacecraft relative to the magnetic reconnection structure observed on 16 October 2015 at 1307 UT. Geophysical Research Letters, 43 , 5589-5596. https://doi.org/10.1002/2016GL069214

Divin, A., Khotyaintsev, Y. V., Vaivads, A., André, M., Toledo-Redondo, S., Markidis, S., \& Lapenta, G. (2016). Three-scale structure of diffusion region in the presence of cold ions. Journal of Geophysical Research: Space Physics, 121, 12,001-12,013. https://doi.org/10.1002/ 2016JA023606

Engwall, E., Eriksson, A. I., Cully, C. M., André, M., Puhl-Quinn, P. A., Vaith, H., \& Torbert, R. (2009). Survey of cold ionospheric outflows in the magnetotail. Annales Geophysicae, 27(8), 3185-3201. https://doi.org/10.5194/angeo-27-3185-2009

Engwall, E., Eriksson, A. I., Cully, C. M., André, M., Torbert, R., \& Vaith, H. (2008). Earth's ionospheric outflow dominated by hidden cold plasma. Nature Geoscience, 2(4), 321-324.

Ergun, R. E., Tucker, S., Westfall, J., Goodrich, K. A., Malaspina, D. M., Summers, D., \& Cully, C. M. (2016). The axial double probe and fields signal processing for the MMS mission. Space Science Reviews, 199(1), 167-188. https://doi.org/10.1007/s11214-014-0115-X

Graham, D. B., Khotyaintsev, Y. V., Norgren, C., Vaivads, A., André, M., Toledo-Redondo, S., et al. (2017). Lower hybrid waves in the ion diffusion and magnetospheric inflow regions. Journal of Geophysical Research: Space Physics, 122, 517-533. https://doi.org/10.1002/ 2016JA023572

Haaland, S., Sonnerup, B. U., Dunlop, M. W., Georgescu, E., Paschmann, G., Klecker, B., \& Vaivads, A. (2004). Orientation and motion of a discontinuity from Cluster curlometer capability: Minimum variance of current density. Geophysical Research Letters, 31 , L10804. https://doi.org/10.1029/2004GL020001

Huddleston, M. M., Chappell, C. R., Delcourt, D. C., Moore, T. E., Giles, B. L., \& Chandler, M. O. (2005). An examination of the process and magnitude of ionospheric plasma supply to the magnetosphere. Journal of Geophysical Research, 110, A12202. https://doi.org/10. 1029/2004JA010401

Ishizawa, A., \& Horiuchi, R. (2005). Suppression of Hall-term effects by gyroviscous cancellation in steady collisionless magnetic reconnection. Physical Review Letters, 95, 45003. https://doi.org/10.1103/PhysRevLett.95.045003

Khotyaintsev, Y. V., Vaivads, A., Retinò, A., André, M., Owen, C., \& Nilsson, H. (2006). Formation of inner structure of a reconnection separatrix region. Physical Review Letters, 97(20), 205003.

Kuznetsova, M. M., Hesse, M., \& Winske, D. (1998). Kinetic quasi-viscous and bulk flow inertia effects in collisionless magnetotail reconnection. Journal of Geophysical Research, 103(A1), 199-213. https://doi.org/10.1029/97JA02699

Le Contel, O., Leroy, P., Roux, A., Coillot, C., Alison, D., Bouabdellah, A., \& de la Porte, B. (2016). The search-coil magnetometer for MMS Space Science Reviews, 199(1), 257-282. https://doi.org/10.1007/s11214-014-0096-9

Li, K., Haaland, S., Eriksson, A., André, M., Engwall, E., Wei, Y., \& Ren, Q. Y. (2013). Transport of cold ions from the polar ionosphere to the plasma sheet. Journal of Geophysical Research: Space Physics, 118, 5467-5477. https://doi.org/10.1002/jgra.50518

Lindqvist, P. A., Olsson, G., Torbert, R. B., King, B., Granoff, M., Rau, D., \& Tucker, S. (2016). The spin-plane double probe electric field instrument for MMS. Space Science Reviews, 199(1), 137-165. https://doi.org/10.1007/s11214-014-0116-9 
Mauk, B., Blake, J., Baker, D., Clemmons, J., Reeves, G., Spence, H. E., et al. (2016). The energetic particle detector (EPD) investigation and the energetic ion spectrometer (EIS) for the Magnetospheric Multiscale (MMS) mission. Space Science Reviews, 199(1-4), $471-514$.

Mozer, F. S., Pritchett, P. L., Bonnell, J., Sundkvist, D., \& Chang, M. T. (2008). Observations and simulations of asymmetric magnetic field reconnection. Journal of Geophysical Research, 113, A00C03. https://doi.org/10.1029/2008JA013535

Nagai, T., Shinohara, I., Fujimoto, M., Hoshino, M., Saito, Y., Machida, S., \& Mukai, T. (2001). Geotail observations of the Hall current system: Evidence of magnetic reconnection in the magnetotail. Journal of Geophysical Research, 106(A11), 25,929-25,949.

Nakamura, R., Varsani, A., Genestreti, K. J., Le Contel, O., Nakamura, T., Baumjohann, W., et al. (2018). Multiscale currents observed by MMS in the flow braking region. Journal of Geophysical Research: Space Physics, 123, 1260-1278. https://doi.org/10.1002/2017JA024686

Pollock, C., Moore, T., Jacques, A., Burch, J., Gliese, U., Saito, Y., \& Zeuch, M. (2016). Fast plasma investigation for magnetospheric multiscale. Space Science Reviews, 199(1), 331-406. https://doi.org/10.1007/s11214-016-0245-4

Pritchett, P. L., \& Mozer, F. S. (2009). Asymmetric magnetic reconnection in the presence of a guide field. Journal of Geophysical Research, 114, A11210. https://doi.org/10.1029/2009JA014343

Retinò, A., Vaivads, A., André, M., Sahraoui, F., Khotyaintsev, Y., Pickett, J., et al. (2006). Structure of the separatrix region close to a magnetic reconnection X-line: Cluster observations. Geophysical Research Letters, 33, L06101. https://doi.org/10.1029/2005GL024650

Robert, P., Dunlop, M. W., Roux, A., \& Chanteur, G. (1998). Accuracy of current density determination. In Analysis methods for multi-spacecraft data (pp. 185-220). Noordwijk: ESA Publications Division.

Russell, C. T., Anderson, B. J., Baumjohann, W., Bromund, K. R., Dearborn, D., Fischer, D., \& Richter, I. (2016). The magnetospheric multiscale magnetometers. Space Science Reviews, 199(1), 189-256. https://doi.org/10.1007/s11214-014-0057-3

Shay, M. A., Drake, J. F., Rogers, B. N., \& Denton, R. E. (2001). Alfévnic collisionless magnetic reconnection and the Hall term. Journal of Geophysical Research, 106(A3), 3759-3772. https://doi.org/10.1029/1999JA001007

Shi, Q. Q., Shen, C., Pu, Z. Y., Dunlop, M. W., Zong, Q. G., Zhang, H., \& Balogh, A. (2005). Dimensional analysis of observed structures using multipoint magnetic field measurements: Application to Cluster. Geophysical Research Letters, 32, L12105. https://doi.org/10.1029/ 2005GL022454

Sonnerup, B. U. Ö. (1979). Magnetic field reconnection. Solar System Plasma Physics, 1, 45-108.

Sonnerup, B. U. Ö., \& Cahill Jr, L. (1967). Magnetopause structure and attitude from Explorer 12 observations. Journal of Geophysical Research, 72(1), 171-183.

Stiles, G. S., Hones Jr, E. W., Bame, S. J., \& Asbridge, J. R. (1978). Plasma sheet pressure anisotropies. Journal of Geophysical Research, 83(A7), 3166-3172. https://doi.org/10.1029/JA083iA07p03166

Toledo-Redondo, S., André, M., Khotyaintsev, Y. V., Lavraud, B., Vaivads, A., Graham, D. B., et al. (2017). Energy budget and mechanisms of cold ion heating in asymmetric magnetic reconnection. Journal of Geophysical Research: Space Physics, 122, 9396-9413. https://doi. org/10.1002/2017JA024553

Toledo-Redondo, S., Vaivads, A., André, M., \& Khotyaintsev, Y. V. (2015). Modification of the Hall physics in magnetic reconnection due to cold ions at the Earth's magnetopause. Geophysical Research Letters, 42, 6146-6154. https://doi.org/10.1002/2015GL065129

Torbert, R. B., Russell, C. T., Magnes, W., Ergun, R. E., Lindqvist, P. A., LeContel, O., et al. (2016). The FIELDS instrument suite on MMS Scientific objectives, measurements, and data products. Space Science Reviews, 199(1-4), 105-135.

Torbert, R. B., Vaith, H., Granoff, M., Widholm, M., Gaidos, J. A., Briggs, B. H., \& Kooi, V. (2016). The electron drift instrument for MMS Space Science Reviews, 199(1), 283-305. https://doi.org/10.1007/s11214-015-0182-7

Vasyliunas, V. M. (1975). Theoretical models of magnetic field line merging. Reviews of Geophysics, 13(1), 303-336.

Young, D. T., Burch, J. L., Gomez, R. G., De Los Santos, A., Miller, G. P., Wilson, P., \& Webster, J. M. (2016). Hot plasma composition analyzer for the magnetospheric multiscale mission. Space Science Reviews, 199(1), 407-470. https://doi.org/10.1007/s11214-014-0119-6 